\title{
High glucose promotes gastric cancer chemoresistance in vivo and in vitro
}

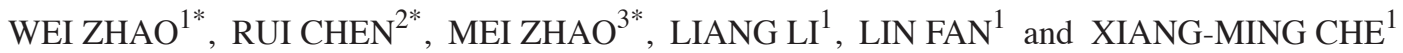 \\ ${ }^{1}$ Department of General Surgery, The First Affiliated Hospital, College of Medicine, Xi'an Jiaotong University, Xi'an, \\ Shaanxi 710061; ${ }^{2}$ Department of Neonatal Surgery, The Children's Hospital, Zhejiang University School of Medicine, \\ Hangzhou, Zhejiang 310003; ${ }^{3}$ Department of Pharmacology, College of Medicine, \\ Xi'an Jiaotong University, Xi'an, Shaanxi 710061, P.R. China
}

Received March 3, 2014; Accepted December 2, 2014

DOI: $10.3892 / \mathrm{mmr} .2015 .3522$

\begin{abstract}
The aim of the present study was to determine whether gastric cancer chemoresistance was increased under high glucose conditions by means of a clinical case study and experimental cytology. The expression of nicotinamide phosphoribosyltransferase (Nampt), silent information regulator 1 (sirt1), p53, p-glycoprotein (P-gp) and topoisomerase (topo)-II $\alpha$ was evaluated in gastric cancer tissues and gastric cancer with diabetes tissues by immunohistochemistry. Subsequently, the survival time of the patients was assessed. For further investigation, the human gastric cancer cell line SGC7901 was subjected to different glucose concentrations and the aforementioned proteins were detected using reverse transcription-quantitative polymerase chain reaction and western blot analysis. Finally, cell sensitivity to chemotherapy treatment was examined in order to elucidate the role of high glucose in MDR. Positive expression of Nampt, Sirt1, p53, P-gp and Topo-II $\alpha$ was observed to be higher in gastric cancer with diabetes patients compared with gastric cancer patients $(\mathrm{P}=0.01,0.003,0.0025,0.016$ and 0.336 , respectively) with reduced survival time. Similar results were observed in SGC7901 cells. Additionally, cell proliferation rates of SGC7901 cells increased at glucose concentrations of 4,500 and 9,000 $\mathrm{mg} / 1$. Notably, the inhibition rates of 5-fluorouracil on cells decreased over $48 \mathrm{~h}$ when treated with 4,500 and $9,000 \mathrm{mg} / 1$ glucose compared with $1,000 \mathrm{mg} / \mathrm{l}$. In conclusion, patients suffering from gastric cancer and diabetes exhibited greater negative effects, such as a poorer response
\end{abstract}

Correspondence to: Professor Xiang-Ming Che, Department of General Surgery, The First Affiliated Hospital, College of Medicine, Xi'an Jiaotong University, 277 Yanta West Road, Xi'an, Shanxi 710061, P.R. China

E-mail: chexiang@mail.xjtu.edu.cn

*Contributed equally

Key words: gastric cancer, diabetes, high glucose, chemoresistance, nicotinamide phosphoribosyltransferase to chemotherapy and had a lower survival time. High glucose conditions promoted gastric cancer cell proliferation and reduced susceptibility to chemotherapy drugs. These data provided a potential diagnostic and therapeutic strategy for gastric cancer chemoresistance.

\section{Introduction}

Gastric cancer is one of the most common types of malignancy, accounting for 8 and $10 \%$, respectively, of malignant tumor total morbidity and mortality (1). Postoperative chemotherapy has been widely utilized following gastric cancer resection (2). However, multidrug resistance (MDR) is often the main cause for the failure of cancer treatment, particularly in gastric cancer (3). In addition, type 2 diabetes has been implicated in carcinogenesis and cancer progression (4). Despite studies investigating the mechanisms underlying the association between type 2 diabetes and cancer development $(5,6)$, there is little information regarding the association between diabetes and MDR (7).

Nicotinamide phosphoribosyltransferase (Nampt) is essential for cell metabolism and survival $(8)$. Bi et al $(9,10)$ demonstrated that the level of Nampt in gastric cancer was higher than in normal tissue and that inhibition of Nampt may enhance the effects of chemotherapy. Nampt overexpression predicted a poor response to doxorubicin-based chemotherapy in patients with breast cancer $(11,12)$. Silent information regulator 1 (Sirt1) is also known to be involved in cell proliferation, differentiation and apoptotic processes. Sirt1 overexpression is associated with a poor prognosis in gastric cancer $(13,14)$. Nampt acts upstream of Sirt1, through the regulation of nicotinamide adenine dinucleotide (NAD) oxidation (15). However, the expression of Nampt and Sirt1 in gastric cancer with diabetes remains to be elucidated.

The aim of the present study was, therefore, to investigate MDR alterations in gastric cancer combined with type 2 diabetes. Subsequently, an in vitro model was established to investigate the role of high glucose in MDR.

\section{Materials and methods}

Chemicals and reagents. D-Glucose and methylthiazolydiphenyl-tetrazolium bromide (MTT) were purchased from 
Sigma-Aldrich (St. Louis, MO, USA). The chemotherapy drug 5-fluorouracil (5-FU) was acquired from The First Affiliated Hospital, College of Medicine, Xi'an Jiaotong University (Xi'an, China). The primary antibodies used were as follows: Rabbit anti-human polyclonal anti-Nampt (AV42255; Sigma-Aldrich), rabbit anti-human polyclonal anti-p53 (sc-126; Santa Cruz Biotechnology,Inc., Santa Cruz, CA, USA)/p-glycoprotein (P-gp; sc-55510; Santa Cruz Biotechnology, Inc.), rabbit anti-human polyclonal anti-topoisomerase (topo)-II $\alpha$ (20233-1-AP; Proteintech, Chicago, IL, USA) and rabbit anti-human monoclonal anti-Sirt1 (sc-15404; Santa Cruz Biotechnology, Inc.). The secondary antibodies and 3,3'-diaminobenzidine were purchased from ZSGB-BIO (Beijing, China).

Patients and samples. A total of 68 patients with gastric cancer and 40 patients with gastric cancer with diabetes, who had undergone radical gastrectomy at The Department of General Surgery, The First Affiliated Hospital, College of Medicine, Xi'an Jiaotong University, between January 2000 and December 2004 were included in the present study. None of the patients had received preoperative neoadjuvant chemotherapy or radiotherapy. According to the tumor node metastasis classification of the International Union Against Cancer, those patients who were in stage I, II or III were matched based on gender and age. The control group was normal paired tissue samples collected from the patients from an area of tissue $>5 \mathrm{~cm}$ away from the tumor. All studies were approved by the Ethics Committee of Xi'an Jiaotong University and every patient provided written informed consent prior to surgery. The baseline data of the study group are shown in Table I.

Cell culture. The human gastric cancer cell line SGC-7901 was provided by the Fourth Military Medical University (Xi'an, China). Cells were cultured in low-glucose Dulbecco's modified Eagle's medium (1,000 mg/l glucose; Invitrogen Life Technologies, Carlsbad, CA, USA) supplemented with $10 \%$ fetal bovine serum (Hangzhou Sijiqing Biological Engineering Materials Co., Ltd., Hangzhou, China) and $100 \mathrm{IU} / \mathrm{ml}$ penicillin and $100 \mu \mathrm{g} / \mathrm{ml}$ streptomycin (Harbin Pharmaceutical Group Co., Ltd, Harbin, China) at $37^{\circ} \mathrm{C}$ in a humidified atmosphere containing $5 \% \mathrm{CO}_{2}$. Cells were subcultured with $0.25 \%$ Trypsin-EDTA (Amresco, LLC, Solon, OH, USA) once they reached $>80 \%$ confluence.

In vitro drug sensitivity assay. For the effective detection of chemotherapy drugs, cells cultured in 1,000, 4,500 and $9,000 \mathrm{mg} / 1$ glucose were subjected to $5-\mathrm{FU}$ at $3.75,15,30$, 60 and $120 \mu \mathrm{g} / \mathrm{ml}$. According to the results of daily cell counting, the growth curve with MTT was produced and the half maximal inhibitory concentration of each group was calculated.

Immunohistochemical staining and scoring. The tissue samples were prepared cutting the paraffin-embedded tissue into serial sections $4 \mu \mathrm{m}$ thick and evenly cut into three sections (one for hematoxylin staining and the other two for immunohistochemical staining). The tissue sections were preserved in $2.5 \%$ glutaraldehyde-polyoxymethylene solution, were dehydrated and embedded in paraffin following routine methods and were immersed in distilled water.
Table I. Characteristics of patients.

\begin{tabular}{lcc}
\hline Characteristic & Gastric cancer & $\begin{array}{c}\text { Gastric cancer } \\
\text { with DM }\end{array}$ \\
\hline Gender & & \\
Male & 50 & 30 \\
Female & 18 & 10 \\
Age (years) & $59.2 \pm 18.4$ & $64.1 \pm 14.6$ \\
Histology & & \\
Differentiated & 56 & 34 \\
Poorly differentiated & 12 & 6 \\
TNM staging & & 11 \\
I+II & 35 & 29 \\
III+IV & 33 & \\
\hline
\end{tabular}

DM, diabetes mellitus; TNM, tumor node metastasis.

Subsequently, the paraffin sections were washed ( 3 x $5 \mathrm{~min})$ in phosphate-buffered saline with Tween-20 (PBS-T; $0.01 \mathrm{M}$; $\mathrm{pH}$ 7.4) and then blocked with $0.3 \% \mathrm{H}_{2} \mathrm{O}_{2}$-methanol at room temperature for endogenous peroxidase ablation. The following steps were conducted in a moist chamber. The non-specific binding sites were blocked by $30-\mathrm{min}$ incubation in 5\% normal goat serum (cat. no. ZLI-9022; Beijing Zhongshan Golden Bridge Company, Beijing, China) at room temperature for $20 \mathrm{~min}$. The primary antibody was then added dropwise, then the sections were incubated for $2 \mathrm{~h}$ or overnight at $4{ }^{\circ} \mathrm{C}$ followed by washing again with in PBS-T ( $3 \times 5 \mathrm{~min}$ ). According to the PV-6000 direction to add the reagent, the sections were incubated for $30 \mathrm{~min}$ at room temperature, followed by washing with PBS-T( $3 \times 5 \mathrm{~min})$. The sections were then stained with 3,3-diaminobenzidine (cat. no. ZLI-9017; Beijing Zhongshan Golden Bridge Company), were kept at room temperature without light for $10 \mathrm{~min}$ and were immersed in distilled water for $10 \mathrm{~min}$. The sections were then stained with hematoxylin for $2 \mathrm{~min}$, completing the staining with the distilled water for $10 \mathrm{~min}$. Dehydration of the sections was then conducted with sequential 1-min ethanol washes starting with $75 \%$, followed by $80 \%$ and finishing with an $100 \%$ ethanol wash. The sections were mounted with neutral gum and then sealed and analyzed by optical microscopy (Nikon 80i; Nikon Corporation, Tokyo, Japan). The intensity of immunostaining was assessed by two different observers, with respect to the staining intensity into the following four grades: -, no staining (negative); +, faintly stained up to $25 \%$ (weak positive);,$++ 25-50 \%$ cells moderately stained (moderate positive);,$+++ 50 \%$ or more cells markedly stained (strong positive) (16).

RNA extraction and reverse transcription quantitative polymerase chain reaction ( $R T-q P C R)$. Total RNA was isolated using the RNA Fast 200 purification kit from Fastagen Biotech (Shanghai, China) according to the manufacturer's instructions. Reverse transcription of RNA was performed with ExScriptTM RT reagent kit (Takara Biotechnology Co., Ltd., Dalian, China). The cDNA was then subjected to 
Table II. Gene sequences for quantitative polymerase chain reaction.

\begin{tabular}{lll}
\hline Human gene & \multicolumn{1}{c}{ Forward primer } & \multicolumn{1}{c}{ Reverse primer } \\
\hline Nampt & 5'-AAGAGACTGCTGGCATAGGA-3' & 5'-ACCACAGATACAGGCACTGA-3' \\
Sirt1 & 5'-TTGTACGACGAAGACGAC-3' & 5'-TAGAGCTTGCATGTGAGG-3' \\
p53 & 5'-TCAGTCTTCCTCCAACC-3' & 5'-GGAAAAGACTGAAGGGTG-3' \\
P-gp & 5'-CATTCCTCCTGGAAATTCAACCT-3' & 5'-CTTCAAGATCCATTCCGACCTC-3' \\
Topo-II $\alpha$ & 5'-TGGAAACAGCCAGTAGAG-3' & 5'-ATCTTTGTCCAGGCTTTG-3' \\
$\beta$-actin & 5'-CCTGGGCATGGAGTCCTGTG-3' & 5'-TCTTCATTGTGCTGGGTGCC-3'
\end{tabular}

Sirt1, silent information regulator 1; Nampt, nicotinamide phosphoribosyltransferase; GC, gastric cancer; P-gp, p-glycoprotein; topo-II $\alpha$, topoisomerase-II $\alpha$.

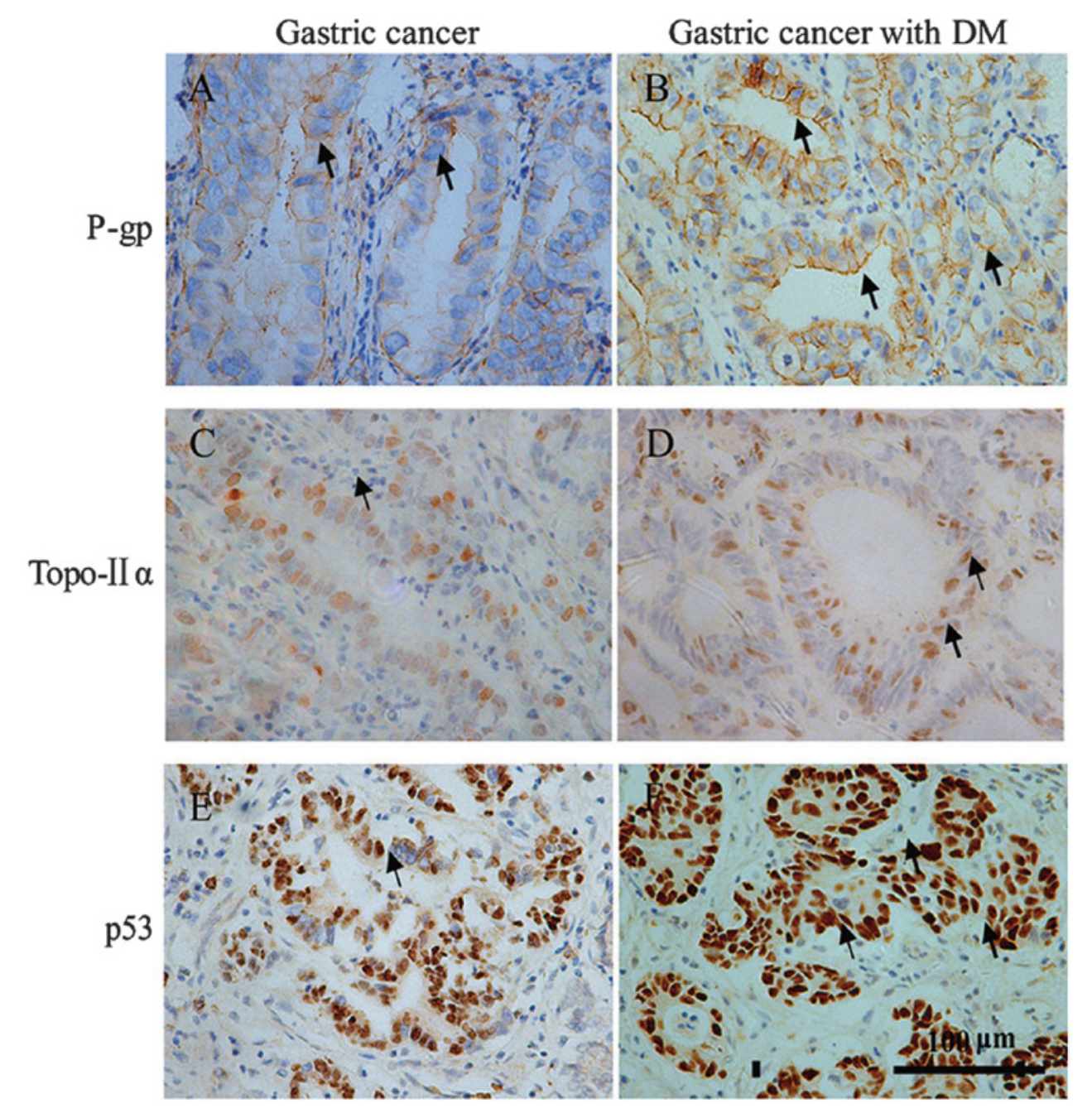

Figure 1. Representative images of immunohistochemical staining with diaminobenzidine and hematoxylin in gastric cancer and gastric cancer with DM. (A and B) P-gp was positive in the cytomembrane. (C and D) Top-II $\alpha$ and (E and F) p53 exhibited nuclear positivity. P-gp, p-glycoprotein; topo-II $\alpha$, topoisomerase-II $\alpha$; DM, diabetes mellitus.

RT-PCR. All primer pairs shown in Table II were designed by Oligo 6.0 primer analysis software (Oswel Research Products, Beijing, China) . qPCR was performed in duplicate reactions of $12.5 \mu 1$ volume SYBR II (Takara Biotechnology Co., Ltd.), $1 \mu$ l each of specific forward and reverse primers and $9.5 \mu \mathrm{l}$ RNase Free $\mathrm{H}_{2} \mathrm{O}$ up to $24 \mu \mathrm{l}$. Samples were heated for $85^{\circ} \mathrm{C}$ followed by 40 cycles of amplification for $15 \mathrm{sec}$ at $95^{\circ} \mathrm{C}$ and $1 \mathrm{~min}$ at $60^{\circ} \mathrm{C}$. The mRNA level of $\beta$-actin was measured as an internal reference.

Protein extraction and western blot analysis. Cells were treated with lysis buffer. Protein samples $(30 \mu \mathrm{g})$ were separated by $10 \%$ gels and then transferred onto a polyvinylidene difluoride membrane (Sterlitech Corp., Kent, WA, USA). 
Table III. Expression of Nampt, Sirt1, p53, P-gp and Top-II $\alpha$ between two groups using $\chi^{2}$ test.

\begin{tabular}{lcc}
\hline Protein & $\begin{array}{c}\text { Positive } \\
\text { cases }\end{array}$ & $\begin{array}{c}\text { Negative } \\
\text { cases }\end{array}$ \\
\hline Nampt & 36 & 32 \\
GC & 31 & 9 \\
GC with diabetes & & \\
Sirt1 & 37 & 31 \\
GC & 33 & 7 \\
GC with diabetes & & \\
p53 & 33 & 35 \\
GC & 29 & 11 \\
GC with diabetes & & 33 \\
P-gp & 35 & 10 \\
GC & 30 & \\
GC with diabetes & & 45 \\
Topo-II $\alpha$ & 23 & 28 \\
GC & 12 & \\
GC with diabetes &
\end{tabular}

Sirt1, silent information regulator 1; Nampt, nicotinamide phosphoribosyltransferase; GC, gastric cancer; P-gp, p-glycoprotein; topo-II $\alpha$, topoisomerase-II $\alpha$.

Blots were probed with antibodies against Nampt $(1: 1,000)$, Sirt1 (1:500), p53 (1:500), P-gp (1:200) and Top-II $\alpha$ (1:200). Following primary antibody incubation, membranes were incubated with horseradish peroxidase-conjugated secondary immunoglobulin $\mathrm{G}(1: 2,000)$. The immunoreaction was visualized using ECL-Plus reagent (Pierce Biotechnology, Inc., Rockford, IL, USA) and analyzed using a Gel-Pro Analyzer (Media Cybernetics, Silver Spring, MD, USA).

Statistical analysis. The data are presented as the mean \pm standard error of the mean. Statistical analysis was performed using SPSS 16.0 software (SPSS, Inc., Chicago, IL, USA). Data were analyzed using a $\chi^{2}$ test. $P<0.05$ was considered to indicate a statistically significant difference. Correlations were analyzed using Spearman's rank correlation coefficient test.

\section{Results}

Alterations in the levels of chemoresistance protein markers in gastric cancer with diabetes. At present, P-gp and p53 are recognized as typical chemoresistance protein markers $(17,18)$. The present study demonstrated increased levels of P-gp and p53 in patients with gastric cancer with diabetes compared with gastric cancer alone (Fig. 1 and Table III). It is well established that Topo-II $\alpha$ is a target for anti-cancer drugs (19). Notably, the level of Topo-II $\alpha$ in gastric cancer with diabetes tissues was lower than that in gastric cancer alone (Fig. 1 and Table III). p53 and Topo-II $\alpha$ were found primarily in the nuclei, while P-gp was identified in the cytomembrane, which is consistent with previous studies $(20,21)$. The clinical pathological features are summarized in Table III.
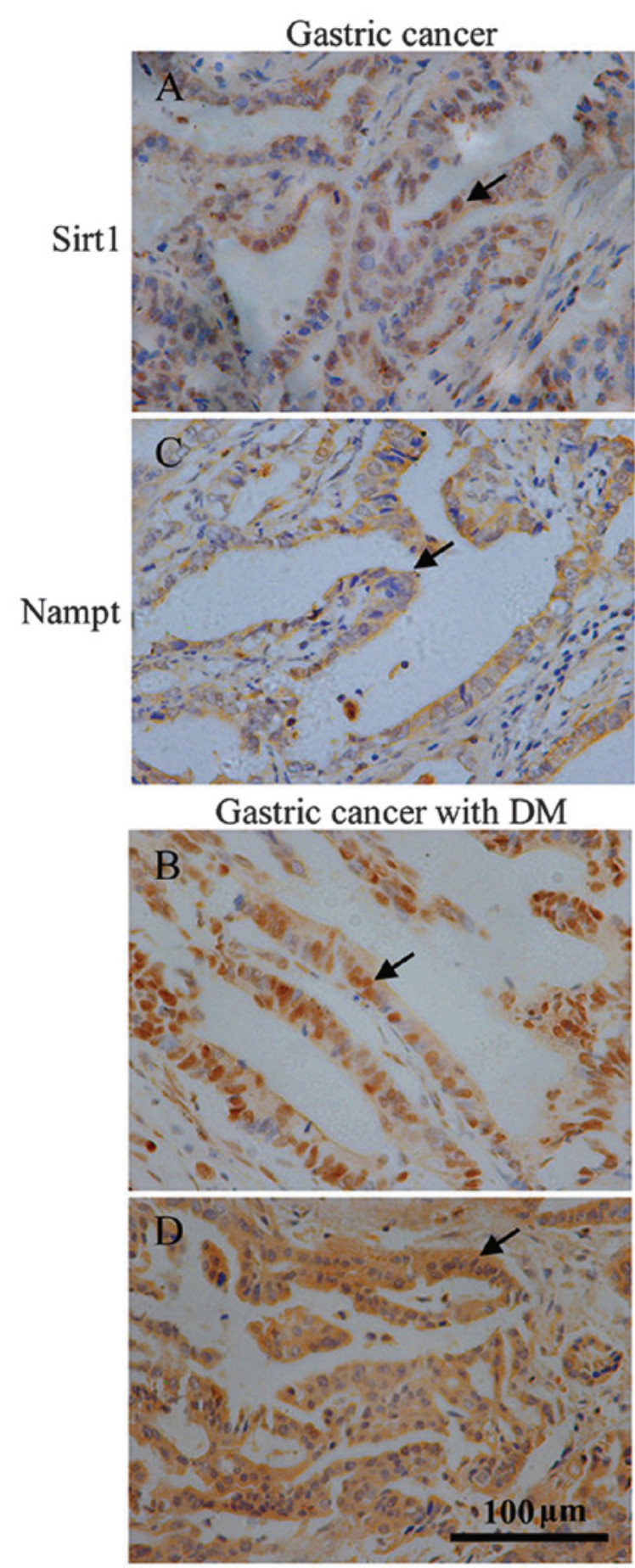

Figure 2. Representative images of immunohistochemical staining with diaminobenzidine and hematoxylin in gastric cancer and gastric cancer with DM. (A and B) Sirt1 exhibited nuclear positivity. (C and D) Nampt exhibited positive staining in the cytoplasm. Sirt1, silent information regulator 1; Nampt, nicotinamide phosphoribosyltransferase; DM, diabetes mellitus.

Diabetes increases the expression of Nampt and Sirtl in gastric cancer and decreases survival rate. To further investigate the mechanism underlying chemoresistance, the present study examined whether Nampt and Sirt1 were involved in the process. Levels of Nampt and Sirt1 were observed to be higher in gastric cancer with diabetes than gastric cancer tissues (Fig. 2 and Table III). To further investigate the impact on survival time, the survival curve was also examined. Increased Nampt was associated with reduced overall survival rate of 
A

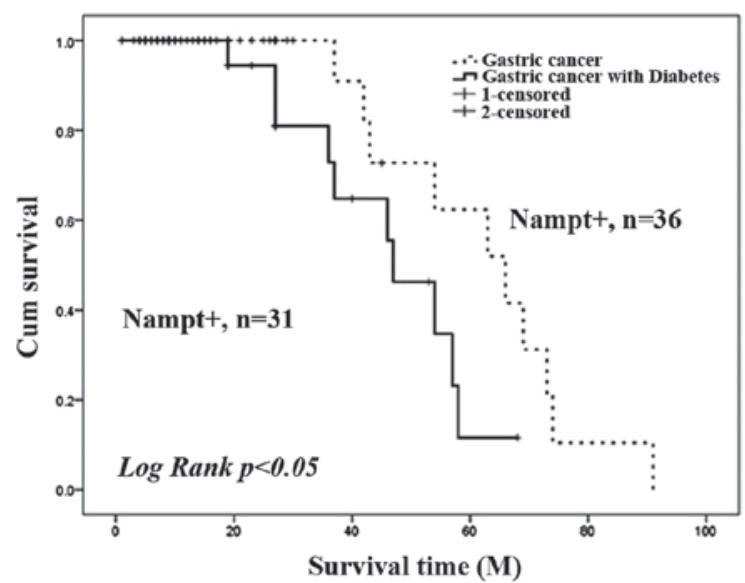

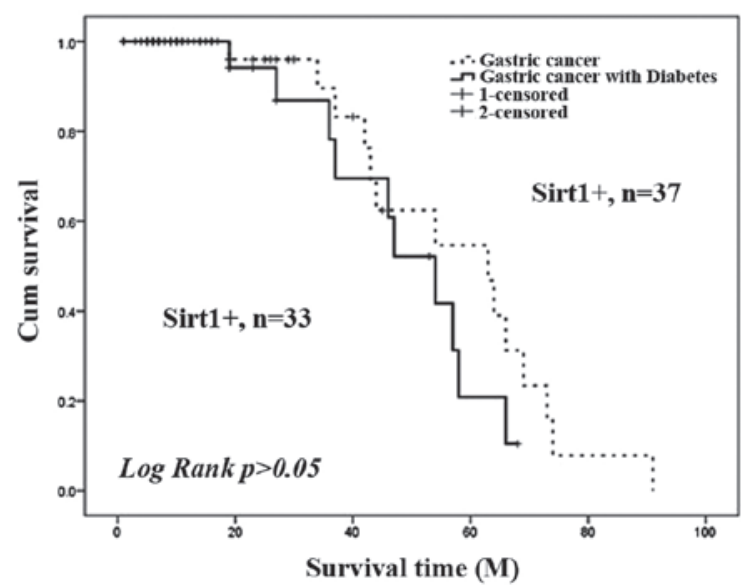

Figure 3. Survival analysis in gastric cancer and gastric cancer with DM. Effects of (A) Nampt and (B) Sirt1 on overall survival rate in gastric cancer and gastric cancer with DM groups. Sirt1, silent information regulator 1; Nampt, nicotinamide phosphoribosyltransferase; DM, diabetes mellitus.

A

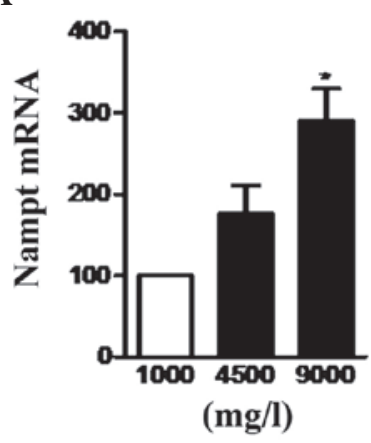

C

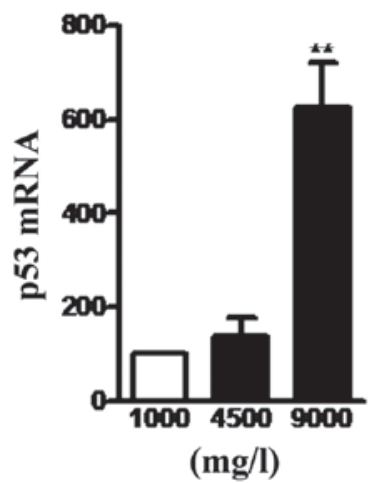

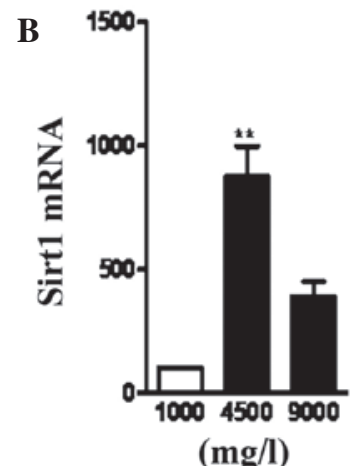

D

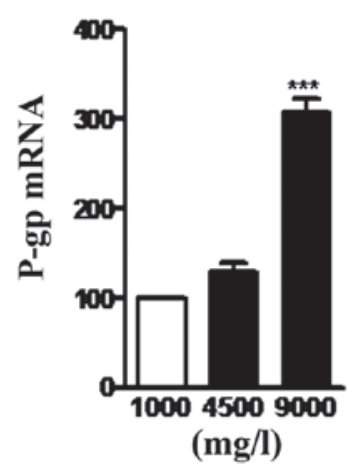

E

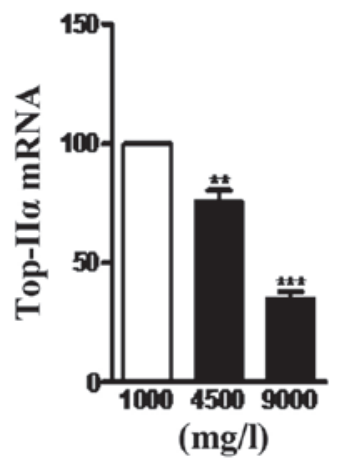

Figure 4. Effect of high glucose on the mRNA expression of Nampt, Sirt1, p53, P-gp and Top-II $\alpha$. SGC7901 cells were plated in 6-well plates and then incubated for $72 \mathrm{~h}$ in the presence of high glucose $(4,500$ and 9,000 $\mathrm{mg} / \mathrm{l})$ in order to examine Nampt, Sirt1, p53, P-gp and Top-II $\alpha$ gene expression using reverse transcription quantitative polymerase chain reaction. Treatment of SGC7901 cells with high glucose resulted in increased (A) Nampt, (B) Sirt1, (C) p53, (D) P-gp and (E) decreased Top-II $\alpha$ mRNA levels. Data are presented as the mean \pm standard error of the mean $(n=6)$. ${ }^{*}<0.05$, ${ }^{* *} \mathrm{P}<0.01$ and ${ }^{* * *} \mathrm{P}<0.001, \mathrm{v} . \mathrm{s}$. the $1,000 \mathrm{mg} / 1$ group. Sirt1, silent information regulator 1; Nampt, nicotinamide phosphoribosyltransferase; P-gp, p-glycoprotein; topo-II $\alpha$, topoisomerase-II $\alpha$.

gastric cancer with diabetes by univariate analysis (Fig. 3). However, Sirt1 had little effect on the overall survival rate (Fig. 3).

High glucose induces the expression of Nampt, Sirt1, p53, $P$-gp and inhibits Top-IIL in the gastric cancer cell line SGC7901. PCR analysis indicated that cells treated with glucose at 4,500 and 9,000 $\mathrm{mg} / \mathrm{l}$ exhibited increased mRNA expression of Nampt, Sirt1, p53 and P-gp compared with $1,000 \mathrm{mg} / \mathrm{l}$. In addition, the levels of Topo-II $\alpha$ were reduced at 4,500 and 9,000 mg/l glucose (Fig. 4). Western blot analysis confirmed these effects at the protein level (Fig. 5). These results suggested that high glucose induced chemoresistance protein markers at the transcriptional and translational levels. 


\section{A}

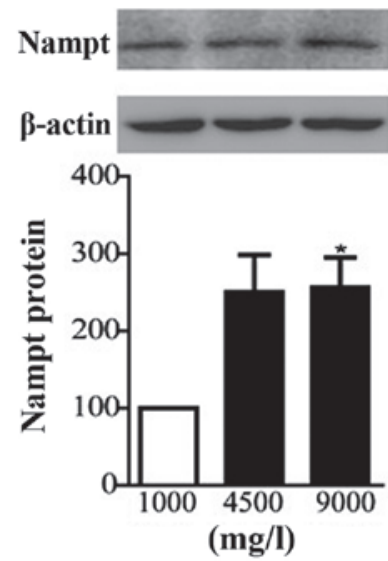

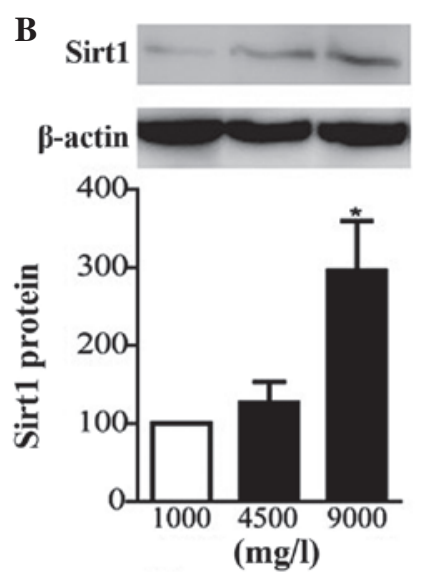

C
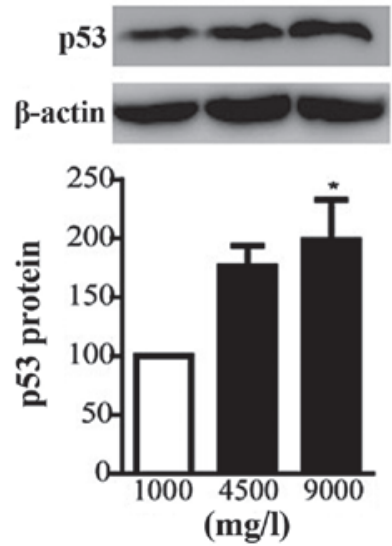

D

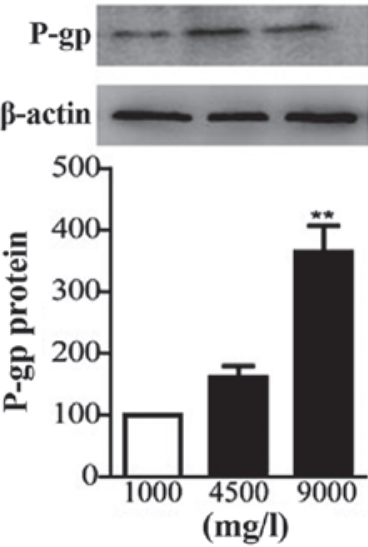

$\mathbf{E}$

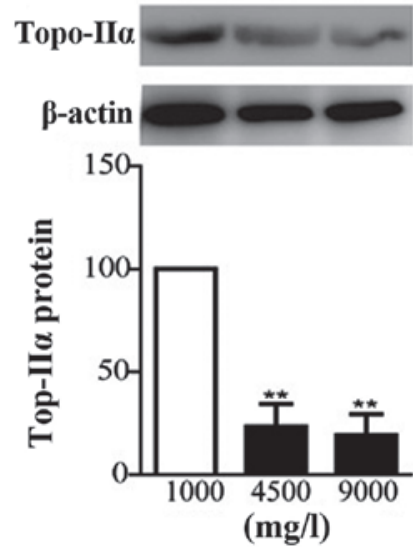

Figure 5. Effect of high glucose on the protein expression of Nampt, Sirt1, p53, P-gp and Top-II $\alpha$. Gastric cancer SGC7901 cells were plated in 6-well plates and incubated for $72 \mathrm{~h}$ in the presence of high glucose $(4,500$ and 9,000 mg/ml) to examine Nampt, Sirt1, p53, P-gp and Top-II $\alpha$ protein expression patterns. Total protein was extracted from cells and analyzed by western blot analysis. The protein expression of (A) Nampt, (B) Sirt1, (C) p53 and (D) P-gp was significantly increased following treatment with $9,000 \mathrm{mg} / \mathrm{ml}$ glucose in SGC7901 cells, however, the expression of (E) Top-II $\alpha$ was significantly decreased. The data are expressed as the mean \pm standard error of the mean $(n=6)$. ${ }^{*} \mathrm{P}<0.05$ and ${ }^{* *} \mathrm{P}<0.01$, high glucose v.s. the $1,000 \mathrm{mg} / 1$ glucose group. Sirt1, silent information regulator 1; Nampt, nicotinamide phosphoribosyltransferase; P-gp, p-glycoprotein; topo-II $\alpha$, topoisomerase-II $\alpha$.

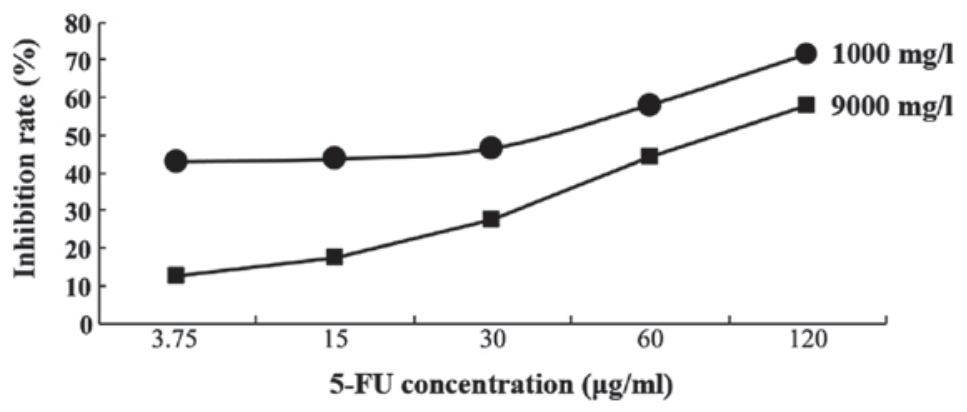

Figure 6. Combination of 5-FU with high glucose increased inhibition of gastric cancer cells. 5-FU, 5-fluorouracil.

High glucose results in chemoresistance of gastric cancer cells treated with 5-FU. Subsequently, it was examined whether high glucose levels induced chemoresistance to 5-FU in SGC-7901 cells. The number of live cells was measured using an MTT assay. When exposed to 5-FU, SGC7901 cells exhibited a higher cell proliferation rate in the presence of high glucose $(9,000 \mathrm{mg} / \mathrm{l})$ compared with lower glucose concentrations $(1,000 \mathrm{mg} / \mathrm{l})$ over $48 \mathrm{~h}$, suggesting that high glucose attenuated the growth inhibitory effect of 5-FU (Fig. 6).

\section{Discussion}

Cancer with type 2 diabetes appears to result in a higher risk of MDR (22). The present study predominantly focused on the effects of high glucose on gastric cancer. Initially, it was identified that chemoresistance protein markers, including P-gp and Topo-II $\alpha$ in diabetes differed from that of cancer alone. Secondly, high glucose increased Nampt and Sirt1, which may provide a possible mechanism for MDR in patients and a lower inhibition rate with anticancer drugs 
in the cancer cell line. The present findings are in line with previous studies concerning the association between diabetes and the prevalence of cancer $(23,24)$.

The wider effects of type 2 diabetes, including hyperglycemia and hyperinsulinemia can lead to cancer progression (25). Based on these studies, a mechanism was postulated to explain the possible promoting impact of diabetes on gastric cancer; hyperglycemia may promote the expression of Nampt/Sirt1 in gastric cancer tissue, thereby upregulating the expression level of mutated p53 in tumor cells. Therefore the possible mechanisms by which MDR occurs may proceed through promoting P-gp expression and reducing Topo-II $\alpha$ expression.

Several studies have observed overexpression of Nampt in gastric cancer and Nampt inhibition has been found to suppress cancer cell growth and induce apoptosis (26). Nampt level in the plasma was higher in the diabetic population compared with healthy controls (27). In line with these studies, in the present study the expression of Nampt in gastric cancer patients with diabetes was found to be higher than that identified in gastric cancer alone. It is well established that Nampt dictates $\mathrm{NAD}^{+}$in mammalian cells and $\mathrm{NAD}^{+}$is central to cell proliferation (28). Nampt promoted cancer cell proliferation through elevated $\mathrm{NAD}^{+}$synthesis. Therefore, Nampt overexpression represents a key mechanism in tumor biology.

Sirt1 is a conserved stress response protein involved in a broad range of biological processes (29). Previous studies have demonstrated that Sirt1 is involved in the development of MDR $(30,31)$. Furthermore, Nampt has been demonstrated to enhance Sirt1 activity by increasing NAD ${ }^{+}(6)$. C-MYC also contributed to Sirt1 activation in breast cancer (32). Other studies have also demonstrated that Sirt1 is important in promoting cell growth and chemoresistance in PC3 and DU145 cells $(33,34)$. Similarly, in the present study it was revealed that Sirt1 expression was upregulated under high glucose conditions. These data indicated that Sirt1 is possibly an import mediator of MDR caused by high glucose and a valuable therapeutic target for gastric cancer.

In order to verify the possible underlying mechanism using cytology, the human gastric cancer cell line SGC7901 was subjected to high glucose medium. Consistent with in vivo results, the hyperglycemic condition promoted the expression of Nampt and Sirt1, then increased the expression of mutated p53 resulting in an upregulation of P-gp and a downregulation of Topo-II $\alpha$. In addition, the high glucose condition promoted gastric cancer cell proliferation and reduced susceptibility to chemotherapy drugs.

Nampt may elevate Sirt1 expression and activity through the glucose metabolic pathway. p53, as a tumor suppressor, is able to induce tumor cell apoptosis and arrest the cell cycle. Sirt1 may inactivate the 382 nd lysine residue of $\mathrm{p} 53$, reduce the expression of the wild-type $\mathrm{p} 53$ protein combining with the DNA cis element to promote $\mathrm{p} 53$ mutation. Compared with that of wild-type p53, the mutated p53 has a longer half-life, which is conductive to observation and evaluation. Overexpression of mutated p53 in tumor cells is positively correlated with higher levels of expression of P-gp, an ATP-dependent protein, resulting in MDR (35).

In conclusion, it was demonstrated that type 2 diabetes conferred MDR to gastric cancer and this effect was at least partially mediated by increased Nampt and Sirt1 expression levels. Thus, in the future, postoperative chemotherapy needs to utilize a combination therapy of gastric cancer treatment and diabetes treatment. In addition, these findings provide novel insights into the role of Nampt and Sirt1 in MDR.

\section{Acknowledgements}

The present study was supported by the Key Project of National Natural Science Foundation of China (grant no. 81172357).

\section{References}

1. Ferlay J, Shin HR, Bray F, et al: Estimates of worldwide burden of cancer in 2008: GLOBOCAN 2008. Int J Cancer 127: 2893-2917, 2010.

2. Hartgrink HH, Jansen EP, van Grieken NC, et al: Gastric cancer Lancet 374: 477-490, 2009.

3. Zhang D and Fan D: New insights into the mechanisms of gastric cancer multidrug resistance and future perspectives. Future Oncol 6: 527-537, 2010.

4. Azoulay L, Yin H, Filion KB, et al: The use of pioglitazone and the risk of bladder cancer in people with type 2 diabetes: nested case-control study. BMJ 344: e3645, 2012.

5. Richardson LC and Pollack LA: Therapy insight: Influence of type 2 diabetes on the development, treatment and outcomes of cancer. Nat Clin Pract Oncol 2: 48-53, 2005.

6. Garten A, Petzold S, Körner A, et al: Nampt: linking NAD biology, metabolism and cancer. Trends Endocrinol Metab 20: 130-138, 2009.

7. Feng Y H, Velazquez-Torres G, Gully C, et al: The impact of type 2 diabetes and antidiabetic drugs on cancer cell growth. J Cell Mol Med 15: 825-836, 2011.

8. Chiarugi A, Dölle C, Felici R, et al: The NAD metabolome - a key determinant of cancer cell biology. Nat Rev Cancer 12: 741-752, 2012.

9. Bi TQ and Che XM: Nampt/PBEF/visfatin and cancer. Cancer Biol Ther 10: 119-125, 2010.

10. Bi TQ, Che XM, Liao XH, et al: Overexpression of Nampt in gastric cancer and chemopotentiating effects of the Nampt inhibitor FK866 in combination with fluorouracil. Oncol Rep 26: 1251-1257, 2011.

11. Burger H, Foekens JA, Look MP, et al: RNA expression of breast cancer resistance protein, lung resistance-related protein, multidrug resistance-associated proteins 1 and 2 , and multidrug resistance gene 1 in breast cancer: correlation with chemotherapeutic response. Clin Cancer Res 9: 827-836, 2003.

12. Bajrami I, Kigozi A, Van Weverwijk A, et al: Synthetic lethality of PARP and NAMPT inhibition in triple-negative breast cancer cells. EMBO Mol Med 4: 1087-1096, 2012.

13. Cha EJ, Noh SJ, Kwon KS, et al: Expression of DBC1 and SIRT1 is associated with poor prognosis of gastric carcinoma. Clin Cancer Res 15: 4453-4459, 2009.

14. Feng AN, Zhang LH, Fan XS, et al: Expression of SIRT1 in gastric cardiac cancer and its clinicopathologic significance. Int J Surg Pathol 19: 743-750, 2011.

15. Imai S: Dissecting systemic control of metabolism and aging in the NAD World: the importance of SIRT1 and NAMPT-mediated NAD biosynthesis. FEBS Lett 585: 1657-1662, 2011.

16. Patel ST, Mistry T, Brown JE, et al. A novel role for the adipokine visfatin/pre-B cell colony-enhancing factor 1 in prostate carcinogenesis. Peptides 31: 51-57, 2010.

17. Tanigawa $\mathrm{N}$ and Fujii $\mathrm{H}$ : Detection of P-glycoprotein and its clinical significance. Nihon Rinsho 55: 1064-1068, 1997 (In Japanese).

18. Ezoe S: Secondary leukemia associated with the anti-cancer agent, etoposide, a topoisomerase II inhibitor. Int J Environ Res Public Health 9: 2444-2453, 2012.

19. Alagoz M, Gilbert DC, El-Khamisy S, et al: DNA repair and resistance to topoisomerase I inhibitors: mechanisms, biomarkers and therapeutic targets. Curr Med Chem 19: 3874-3885, 2012.

20. Karim S and Ali A: Correlation of p53 over-expression and alteration in $\mathrm{p} 53$ gene detected by polymerase chain reaction-single strand conformation polymorphism in adenocarcinoma of gastric cancer patients from India. World J Gastroenterol 15: 1381-1387, 2009.

21. Hollstein M, Sidransky D, Vogelstein B and Harris CC: p53 mutations in human cancers. Science 253: 49-53, 1991. 
22. Renehan AG, Yeh HC, Johnson HA, Wild SH, Gale EA, and Møller H; Diabetes and Cancer Research Consortium: Diabetes and cancer (2): evaluating the impact of diabetes on mortality in patients with cancer. Diabetologia 55: 1619-1632, 2012.

23. Colmers IN, Bowker SL, Tjosvold LA, et al: Insulin use and cancer risk in patients with type 2 diabetes: a systematic review and meta-analysis of observational studies. Diabetes Metab 38 485-506, 2012.

24. Vigneri P, Frasca F, Sciacca L, Pandini G and Vigneri R: Diabetes and cancer. Endocr Relat Cancer 16: 1103-1123, 2009.

25. Shikata K, Ninomiya $T$ and Kiyohara Y: Diabetes mellitus and cancer risk: review of the epidemiological evidence. Cancer Sci 104: 9-14, 2013.

26. Li HJ, Che XM, Zhao W, et al: Diet-induced obesity potentiates the growth of gastric cancer in mice. Exp Ther Med 4: 615-620, 2012.

27. Paschou P, Kukuvitis A, Yavropoulou MP, et al: Genetic variation in the visfatin (PBEF1/NAMPT) gene and type 2 diabetes in the Greek population. Cytokine 51: 25-27, 2010.

28. Imai S: The NAD World: a new systemic regulatory network for metabolism and aging - Sirt1, systemic NAD biosynthesis, and their importance. Cell Biochem Biophys 53: 65-74, 2009.

29. Yi J and Luo J: SIRT1 and p53, effect on cancer, senescence and beyond. Biochim Biophys Acta 1804: 1684-1689, 2010.
30. Zhu H, Xia L, Zhang Y, et al: Activating transcription factor 4 confers a multidrug resistance phenotype to gastric cancer cells through transactivation of SIRT1 expression. PLoS One 7: e31431, 2012

31. Liang XJ, Finkel T, Shen DW, Yin JJ, Aszalos A and Gottesman MM: SIRT1 contributes in part to cisplatin resistance in cancer cells by altering mitochondrial metabolism. Mol Cancer Res 6: 1499-1506, 2008.

32. Menssen A, Hydbring P, Kapelle K, et al: The c-MYC oncoprotein, the NAMPT enzyme, the SIRT1-inhibitor DBC1, and the SIRT1 deacetylase form a positive feedback loop. Proc Natl Acad Sci USA 109: E187-E196, 2012.

33. Kojima K, Ohhashi R, Fujita Y, et al: A role for SIRT1 in cell growth and chemoresistance in prostate cancer PC 3 and DU145 cells. Biochem Biophys Res Commun 373: 423-428, 2008.

34. Fujita Y, Kojima K, Hamada N, et al: Effects of miR-34a on cell growth and chemoresistance in prostate cancer PC3 cells. Biochem Biophys Res Commun 377: 114-119, 2008.

35. de Figueiredo-Pontes LL, Pintão MC, Oliveira LC, et al: Determination of P-glycoprotein, MDR-related protein 1, breast cancer resistance protein, and lung-resistance protein expression in leukemic stem cells of acute myeloid leukemia. Cytometry B Clin Cytom 74: 163-168, 2008. 